\title{
Cytokine storm in severe patient COVID-19 and curative plasmapheresis
}

\author{
Victoria Rusu \\ Department of Anesthesiology and Resuscitation No 2, Nicolae Testemitanu State University of Medicine \\ and Pharmacy Chisinau, the Republic of Moldova
}

Author's ORCID iD, academic degrees and contribution are available at the end of the article

Corresponding author - Victoria Rusu, e-mail: victoria_rusu@mail.ru

Manuscript received July 26, 2021; revised manuscript August 18, 2021; published online September 10, 2021

\begin{abstract}
Background: In the intensive care unit, the curative plasmapheresis technique is widely applied among severe patients, who show signs of both endogenous and exogenous intoxication. The impact of isovolemic isooncotic curative plasmapheresis on the status of hemoleukogram and immunological markers has not been studied yet.

Material and methods: The study is retrospective, observational. The medical records of 34 COVID-19 patients from the COVID intensive care units of Timofei Mosneaga Republican Clinical Hospital who benefited from isovolemic, curative isooncotic plasmapheresis were analyzed. There were 25 complete, analyzed data sets. General data were recorded: preplasmapheresis, postplasmaphesis hemoleukogram.

Results: Isovolemic, isooncotic plasmapheresis, performed in patients with severe COVID-19, SARS-CoV2 showed effects of improving the status of laboratory indices that denote the evolution and favorable prognosis of the disease. The activity of the cytokine storm decreased in both groups of patients, both in those with a mean age of up to 60 years and in those over 60 years of age, with $p=0.001$. Patients over 60 years of age had a marked improvement in serum ferritin index, compared to patients in the age group up to 60 years, in the period after plasmapheresis, $p=0.043$. Analogously, the level of C-reactive protein was analyzed, which clearly decreases in patients $<60$ years of age, compared to those $>60$ years of age, $\mathrm{p}=0.037$

Conclusions: Isovolemic, isooncotic plasmapheresis is a procedure performed in severe COVID-19 (SARS-CoV-2) patients to improve the status of the cytokine storm. Moreover, the performance of the procedure in different age groups induced an improvement in the satiety of hemoleukogram and proinflammatory indices.

Key words: plasmapheresis, cytokine storm, immune status, hemoleukogram.
\end{abstract}

\section{Cite this article}

Rusu V. Cytokine storm in severe patient COVID-19 and curative plasmapheresis. Mold Med J. 2021;64(3):32-35. https://doi.org/10.52418/moldovanmed-j.64-3.21.06.

\section{Introduction}

Curative plasmapheresis is a method of related therapy applied in different pathological conditions in order to remove from the body pathological products of different genesis [1]. The mechanism of elimination and biotransformation of the pathological substrate is the key to the detoxification effect. The correction of the rheological status is achieved by the mechanism of decreasing blood viscosity, increasing plasticity and decreasing the aggregation of blood cells [2]. Immunocorrection is due to the elimination of immune complexes, cytokines, immunocompetent cells thus achieving the "unblocking" of the immune system [3]. In the intensive care unit, the technique of curative plasmapheresis is widely applied among severe patients, who show signs of both endogenous and exogenous intoxication [4].

COVID-19 is a systemic pathology with a significant impact on the hematopoietic system and the immune system $[6,8,9,14]$. Lymphopenia can be considered a laboratory indicator of major significance for the prognosis of COVID-19 patients with the evaluation of serum ferritin. The neutrophil / lymphocyte ratio and the maximum platelet / lymphocyte ratio may also have prognostic value in determining severe cases [3].

Some authors recommend close monitoring of leukocyte count, lymphocyte count, platelet count, and serum ferritin as markers for determining the potential prognosis for a critical disease $[12,13]$.

No references were found to the impact of plasmapheresis on the status of the hemoleukogram and the cytokine storm in COVID-19 (SARS-CoV-2) patients in the intensive care unit. In this regard, the aim of the study was to evaluate the status of hemoleukogram and proinflammatory markers in severe patients in the Intensive Care Unit with the diagnosis of COVID-19 [7, 1011$]$.

\section{Material and methods}

The study is retrospective, observational. The medical records of 34 COVID-19 patients from the COVID intensive care units of Timofei Mosneaga Republican Clinical Hospital who benefited from isovolemic, curative isooncotic plasmapheresis were analyzed. Duration of data collection: April-May 2020. The participants eligible to be included 
in the study were 34 patients. There were 25 patients with complete data sets containing fully analyzed data sheets.

The indications for the plasmapheresis procedure were: elevated values of serum ferritin, C-reactive protein, lymphocytopenia.

The inclusion criteria in the study were:

- Adult patient ( $\geq 18$ years), beneficiary of curative plasmapheresis;

- Patient diagnosed with COVID-19 in intensive care.

The exclusion criteria from the study were:

- Incomplete standardized data sheet.

Statistical analysis

The numerical values of the parameters were numbered in the Excel table, after which - imported into the statistical analysis software GraphPad Prism version 8. The data are presented in the form of absolute and relative values, or average and interval $95 \%$ reliable average. Applied statistical tests: One way ANOVA, Simple T test. P $<0.05$ was considered statistically significant.

\section{Results}

The dynamics of the laboratory parameters, measured before and after the plasmapheresis session is presented in table 1.

Analysis of hemoleukogram data and proinflammatory markers up to plasmapheresis vs the period after plasmapheresis, in patients up to 60 years of age and over 60 years, shows in both age groups a positive dynamics of the results of laboratory indices. Of note is the result for serum ferritin, which in the general group shows a significant decrease in mean values with $\mathrm{n}=1093.7$ to $\mathrm{n}=564.1$.

The results shown in table 1 demonstrate an improve- ment in immune status, with an increase in the number of lymphocytes in the whole group from $7 \%$ to $12.69 \%$ and in age groups from $9 \%$ to $15 \%$ (over 60 years), from $6 \%$ to $11 \%$ (under 60 years).

\section{Discussion}

The activity of the cytokine storm decreased in both groups of patients: with an average age of up to 60 years and in over 60 years of age.

In the series of data with values of laboratory parameters, it was observed that in some patients the results of the analyses decreased faster, and in others - slower. Thus, the results were analyzed in patients depending on the presence of comorbidities, which according to the literature influence the severity prognosis of COVID-19, SARS-CoV2.

Bolagholi S. et al. [4] noticed that plasmapheresis has a beneficial impact on prognosis of COVID-19, but there is no evidence about the influence of the comorbidities on the dynamics of the process.

Patients in the study who had concomitant diabetes mellitus, the outcome of hemoleukogram indices and proinflammatory markers were evaluated to determine the influence of concomitant pathology on plasmapheresis efficacy. Blood glucose values were not investigated in the patients included in the study. The patient with diabetes mellitus was evaluated as a criterion.

The number of leukocytes, C-reactive protein, serum ferritin, and lymphocytes in patients with diabetes mellitus after isovolemic isooncotic plasmapheresis, while maintaining hemodynamic and respiratory protective status, were evaluated and the result showed an improvement in this proinflammatory status of patients after plasmapheresis.

Table 1. Dynamics of laboratory parameters before and after isovolemic isooncotic plasmapheresis

\begin{tabular}{|c|c|c|c|c|}
\hline Parameter & Preplasmapheresis & Postplasmapheresis & $\mathrm{t}$ & p \\
\hline & $(n=)$ & $(\mathrm{N}=)$ & & \\
\hline PCR (whole batch) mg/dl & 118.91 & 55.3 & 2.11 & 0.0001 \\
\hline PCR sublot over 60 years & 135.71 & 38.13 & 1.451 & 0.0011 \\
\hline PCR sublot under 60 years & 108.41 & 66.04 & 2.34 & 0.005 \\
\hline Leukocytes (whole group) & 13.86 & 13.16 & 3.4 & 0.0001 \\
\hline Leukocytes sublot over 60 years & 11.49 & 13.02 & 3.43 & 0.0005 \\
\hline Leukocytes sublot under 60 years & 15.34 & 13.26 & 3.91 & 0.0017 \\
\hline Lymph (whole group) & $7 \%$ & $12.69 \%$ & 1.41 & 0.0001 \\
\hline Lymph sublot over 60 years & $9 \%$ & $15 \%$ & 1.29 & 0.001 \\
\hline Lymph sublot under 60 years & $6 \%$ & $11 \%$ & 1.52 & 0.0003 \\
\hline Monocytes (whole lot) & 4.08 & 3.25 & 1.61 & 0.0001 \\
\hline Monocytes sublot over 60 years & 3.42 & 3.2 & 1.84 & 0.0467 \\
\hline Monocytes sublot under 60 years & 4.08 & 3.2 & 1.65 & 0.0002 \\
\hline Ferritin (whole lot) & 1093.73 & 765.35 & 2.35 & 0.0001 \\
\hline Ferritin it sublot over 60 years & 613.08 & 564.1 & 2.27 & 0.04 \\
\hline Ferritin sublot under 60 years & 1394.15 & 891.13 & 2.498 & 0.0005 \\
\hline
\end{tabular}


Similarly, the patients in the study who had concomitant hypertension were analyzed from a statistical point of view, which according to previous research is also a reserved prognostic indicator in case of severe evolution of COVID-19.

During the research, the results of hemoleukogram indices and proinflammatory markers were analyzed in patients with concomitant hypertension for determining the degree of influence of the concomitant condition on the efficacy of plasmapheresis. The T-student test presented a statistically significant result with $\mathrm{p}=0.045$, with the improvement of the laboratory indicators analyzed among patients with hypertension, although the evolution of the dynamics of laboratory analyses is noticeable to be slow. Similar to the group of patients with diabetes, no different blood pressure values were analyzed, and the results of the indices after performing the plasmapheresis procedure. Reference value showed the presence or absence of pathology of hypertension in the patient. There was a positive evolution of proinflammatory markers and hemoleukogram in this group of patients, significant both clinically and statistically.

In the research, similar to other subjects, it was determined that the age of over 60 increases the predictability of mortality and morbidity for COVID-19. As reference markers of the cytokine storm in the study, the following were selected: C-reactive protein, serum ferritin and lymphocytes. Thus, one of the objectives was to analyze the correlation of different age groups with the evolution of the above-mentioned indices at the stage of pre-plasmapheresis and post-plasmapheresis for establishing the existence of the correlation between the plasmapheresis procedure and the results of the laboratory indices.

Rodríguez-Morales A., Cardona-Ospina J. et al. [5] in their meta-analyses determined that the impact of comorbidities on the prognostic of the COVID-19, in ICU patients is huge, and the damage as well. This study brings the high-light of a procedure that may improve the outcome in that group of patients. More studies are needed to be continued for better analyses.

Thus, four groups of patients were statistically analyzed. The indicator analyzed was the value of C-reactive protein. There is a decrease in C-reactive protein levels in postplasmapheresis patients, both in those up to 60 years of age and after 60 years. It is also worth noting that the level of C-reactive protein clearly decreases in patients $<60$ years of age, compared to those $>60$ years of age. Although among those of $>60$ years a positive dynamics is maintained at the stage after plasmapheresis.

The represented analysis is statistically significant, with $\mathrm{p}$ value $=0.037$. And the improvement of this marker for both age groups after performing the isooncotic, isovolemic plasmapheresis procedure emphasizes the usefulness of the procedure itself in COVID-19 patients in the intensive care unit.
Patients over 60 years of age showed a marked improvement in indices compared to patients in the age group up to 60 years, in the period after plasmapheresis. At the same time, the study demonstrates the efficiency of achieving isovolemic isooncotic plasmapheresis among patients with different age groups. The analysis has statistical significance, with $\mathrm{p}$ value $=0.041$. The result is all the more clinically significant as it correlates with the C-reactive protein indicator, and the hemodynamic and respiratory stability of patients during and after the plasmapheresis procedure.

\section{Conclusions}

Isovolemic, isooncotic plasmapheresis is a procedure performed in severe COVID-19 (SARS-CoV-2) patients to improve the status of the "cytokine storm". Moreover, performing the procedure in different age groups induced an improvement in the satiety of hemoleukogram and proinflammatory indices. And the presence of concomitant diseases did not influence the effectiveness of the results after the realization of isovolemic, isooncotic plasmapheresis.

\section{References}

1. Zhou B, She J, Wang Y, Ma X. Utility of Ferritin, Procalcitonin, and C-reactive Protein in severe patients with 2019 Novel Coronavirus Disease. ResearchSquare. 2020. Epub 2020 April. doi: 10.21203/rs.3. rs-23645/v1.

2. Chen N, Zhou M, Dong X, et al. Epidemiological and clinical characteristics of 99 cases of 2019 novel coronavirus pneumonia in Wuhan, China: a descriptive study. Lancet. 2020 Feb 15;395(10223):507-513. doi: 10.1016/S0140-6736(20)30211-7.

3. Lalueza A, Ayuso B, Arrieta E, et al. Elevation of serum ferritin levels for predicting a poor outcome in hospitalized patients with influenza infection. Clin Microbiol Infect. 2020;26(11):1557.e9-1557.e15. doi: 10.1016/j.cmi.2020.02.018.

4. Balagholi S, Dabbaghi R, Eshghi P, et al. Potential of therapeutic plasmapheresis in treatment of COVID-19 patients: Immunopathogenesis and coagulopathy. Transfus Apher Sci. 2020;59(6):102993. doi: https:// doi.org/10.1016/j.transci.2020.102993.

5. Rodríguez-Morales A, Cardona-Ospina J, Gutiérrez-Ocampo E, et al. Clinical, laboratory and imaging features of COVID-19: a systematic review and meta-analysis. Travel Med Infect Dis. 2020;34:101623. doi: 10.1016/j.tmaid.2020.101623.

6. Wang D, Hu B, Hu C, et al. Clinical characteristics of 138 hospitalized patients with 2019 Novel Coronavirus-infected pneumonia in Wuhan, China. JAMA. 2020;323(11):1061-1069. doi: 10.1001/jama.2020.1585.

7. Senjo H, Higuchi T, Okada S, Takahashi O. Hyperferritinemia: causes and significance in a general hospital. Hematology. 2018;23(10):817822. doi: 10.1080/10245332.2018.1488569.

8. Liu Y, Gayle A, Wilder-Smith A, Rocklov J. The reproductive number of COVID-19 is higher compared to SARS coronavirus. J Travel Med. 2020 Mar 13;27(2):taaa021. doi: 10.1093/jtm/taaa021.

9. Wu F, Zhao S, Yu B. A new coronavirus associated with human respiratory disease in China. Nature. 2020;579(7798):265-269. doi: 10.1038/ s41586-020-2008-3.

10. Slaats J, Ten Oever J, van de Veerdonk F, Netea M. IL-1beta/IL-6/CRP and IL-18/ferritin: distinct inflammatory programs in infections. PLoS Pathog. 2016;12(12):e1005973. doi: 10.1371/journal.ppat.1005973.

11. Kernan K, Carcillo J. Hyperferritinemia and inflammation. Int Immunol. 2017;29(9):401-409. doi: 10.1093/intimm/dxx031.

12. Sanaei Dashti A., Alizadeh S., Karimi A, et al. Diagnostic value of lactate, procalcitonin, ferritin, serum-C-reactive protein, and other biomarkers in bacterial and viral meningitis: a cross-sectional study. Medicine (Baltimore). 2017;96(35):e7637. doi: 10.1097/MD.0000000000007637. 
13. Kawamata R, Yokoyama K, Sato M, et al. Utility of serum ferritin and lactate dehydrogenase as surrogate markers for steroid therapy for Mycoplasma pneumonia. J Infect Chemother. 2015;21(11):783-9. doi: 10.1016/j.jiac.2015.07.009
14. Eurosurveillance Editorial Team. Note from the editors: World Health Organization declares novel coronavirus (2019-nCoV) sixth public health emergency of international concern. Euro Surveill. 2020;25(5):200131e. doi: 10.2807/1560-7917.ES.2020.25.5.200131e.

\section{Author's ORCID iD and academic degrees}

Victoria Rusu, MD, PhD, Assistant Professor of Anesthesiology - https://orcid.org/0000-0002-5241-4067

\section{Author's contribution}

VR conceptualized the idea, conducted literature review, collected the data, interpreted the data, and wrote the manuscript.

\section{Funding}

This study was supported by Nicolae Testemitanu State University of Medicine and Pharmacy. The trial was the author's initiative. The author is independent and takes responsibility for the integrity of the data and accuracy of the data analysis.

\section{Ethics approval and consent to participate}

The study was approved by the Research Ethics Committee of Nicolae Testemitanu State University of Medicine and Pharmacy (Protocol No 28 of 24.02.2015). An informed consent from all participants in the study was obtained.

\section{Conflict of Interests}

There is no known conflict of interests to declare. 